\title{
Teste da ibopamina tópica em pacientes normais, suspeitos e portadores de glaucoma
}

\section{Topic ibopamine test in normal, suspects and glaucoma patients}

Marina Lotto Cordeiro', Walter Amorim², Jose Ricardo Carvalho Lima Rehder ${ }^{3}$

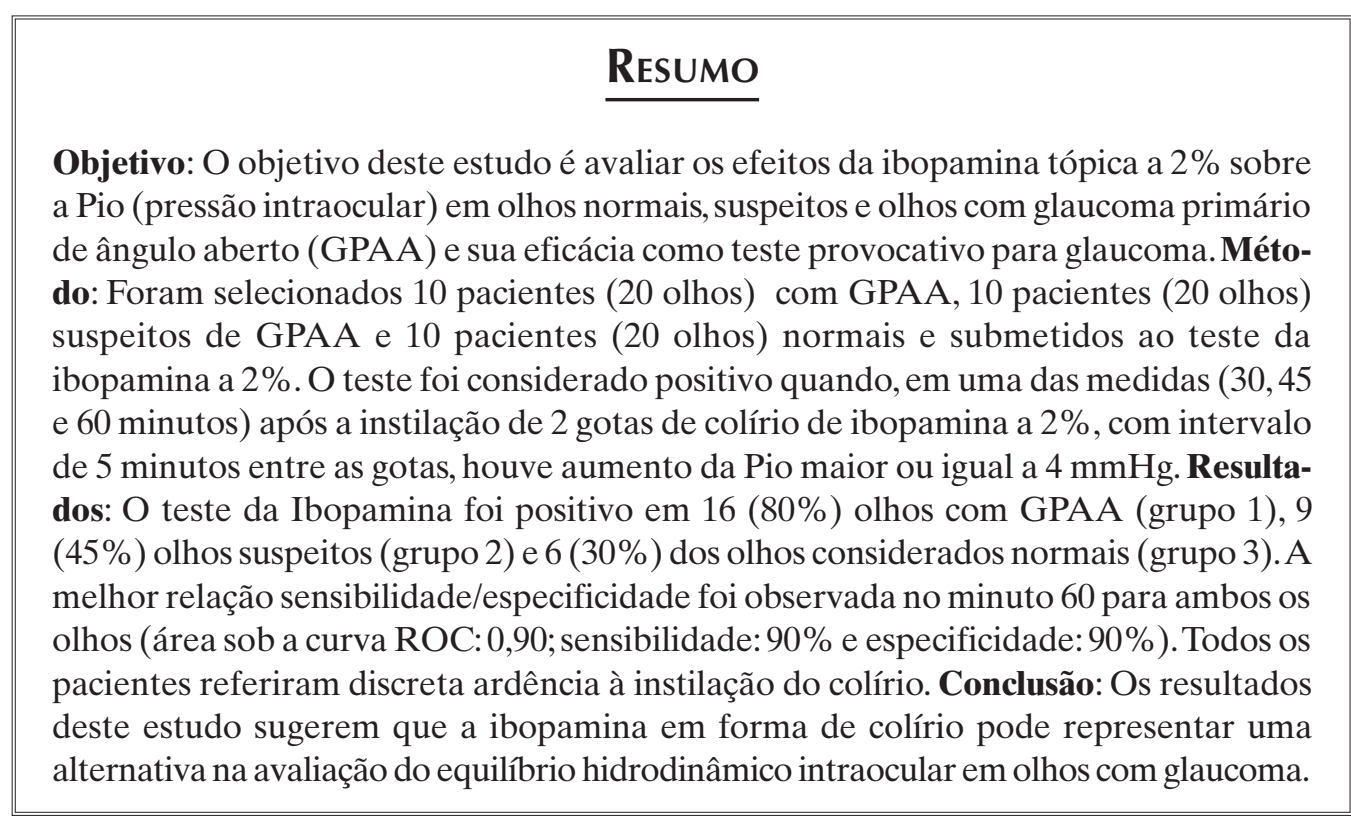

Descritores: Agonistas dopaminérgicos/administração \& dosagem; Glaucoma de ângulo aberto/diagnóstico; Pressão intraocular/efeito de drogas; Soluções oftálmicas; Sensibilidade e especificidade

\footnotetext{
${ }^{1}$ Residente do $1^{\circ}$ ano do Departamento de Oftalmologia da Faculdade de Medicina do ABC - FMABC - Santo André (SP), Brasil; ${ }^{2}$ Chefe do Setor de Glaucoma do Departamento de Oftalmologia da Faculdade de Medicina do ABC - FMABC - Santo André (SP), Brasil;

${ }^{3}$ Professor Titular do Departamento de Oftalmologia da Faculdade de Medicina do ABC - FMABC - Santo André (SP), Brasil;

Trabalho realizado no Departamento de Oftalmologia da Faculdade de Medicina do ABC - FMABC - Santo André (SP), Brasil.

Recebido para publicação em: 18/8/2009 - Aceito para publicação em 10/9/2009
} 


\section{INTRODUÇÃO}

A ibopamina (3,4 diisobutirilester de N-metildopamina) é uma droga simpatomimética dopaminérgica periférica que aumenta o fluxo sanguíneo renal e é utilizada, por via oral, no tratamento da insuficiência cardíaca congestiva.

$\mathrm{Na}$ forma tópica ocular exerce sobre o olho ação midriática, não-cicloplégica, e ativa os receptores D1 dopaminérgicos presentes no epitélio secretor não pigmentado do corpo ciliar, sendo capaz de induzir aumento da pressão intraocular (Pio) em olhos com vias de escoamento do humor aquoso alteradas ${ }^{(1,2)}$.

O efeito hipertensivo da ibopamina tópica é transitório (150 a 180 minutos) e não está relacionado à midríase e sim, ao aumento da produção de humor aquo$\mathrm{so}^{(3-6)}$.

O objetivo deste estudo é avaliar os efeitos da ibopamina tópica a $2 \%$ sobre a Pio em olhos normais, suspeitos e olhos com glaucoma primário de ângulo aberto e sua eficácia como teste provocativo para glaucoma.

\section{Métodos}

\section{Design do estudo e população}

A coleta de dados foi realizada no Departamento de Glaucoma da Disciplina de Oftalmologia da Faculdade de Medicina do ABC.

Foram examinados 30 pacientes ( 60 olhos) e distribuídos em 3 grupos distintos, de acordo com os critérios de inclusão previamente estabelecidos.

Do total de pacientes, 13 eram do sexo masculino e 17 do sexo feminino. A média de idade foi de 58 anos, variando entre 46 e 78 anos.

Os olhos foram divididos em 3 grupos de 10 pacientes, que apresentaram as seguintes características:

Grupo 1:20 olhos de 10 pacientes, 5 do sexo masculino e 5 do sexo feminino; idade média de 62 anos (variando entre 45 e 78 anos), com o diagnóstico de glaucoma primário de ângulo aberto, de acordo com os seguintes critérios:

- Seio camerular aberto, evidenciado pela gonioscopia (desde Linha de Shwalb até Esporão Escleral visível em todos os olhos);

- Fundoscopia, mostrando ao exame do disco óptico, alterações características de glaucoma;

- Perimetria computadorizada Humphrey, Threshold Test, Central 24-2, convencional, apresentando alteração característica para glaucoma e correspondente à fundoscopia;
- Olhos não submetidos a procedimentos cirúrgicos incisionais ou laser;

- Pio detectada $>21 \mathrm{mmHg}$;

- Excluídos pacientes utilizando análogos de prostaglandina para o tratamento de glaucoma.

Grupo 2: 20 olhos, de 10 pacientes, 4 do sexo masculino e 6 do sexo feminino; idade média de 51 anos (variando entre 38 e 72 anos), com a suspeita de glaucoma primário de ângulo aberto (hipertensos oculares), de acordo com os seguintes critérios:

- Fundoscopia, mostrando ao exame do disco óptico, alterações características de glaucoma;

- Perimetria computadorizada Humphrey, Threshold Test, Central 24-2, convencional, normal;

- Pio detectada $>21 \mathrm{mmHg}$.

- Olhos não submetidos a procedimentos cirúrgicos incisionais ou laser;

Grupo 3: 20 olhos, de 10 pacientes, 4 do sexo masculino e 6 do sexo feminino; idade média de 61 anos (variando entre 46 e 78 anos), de acordo com os seguintes critérios:

- Pio detectada menor que $21 \mathrm{mmHg}$;

- Perimetria computadorizada Humphrey, Threshold Test, Central 24-2, convencional, normal;

- Seio camerular aberto evidenciado pela gonioscopia;

- Fundoscopia normal;

- Olhos não submetidos a procedimentos cirúrgicos incisionais ou laser.

Coleta de dados

Todos os pacientes foram submetidos ao teste da ibopamina a $2 \%$ seguindo a sequência:

1.Tonometria de aplanação com tonômetro de Goldman;

2.Instilação de 1 gota de colírio - ibopamina a $2 \%$;

3.Intervalo de 5 minutos;

4.Instilação de 1 gota de colírio - ibopamina a $2 \%$;

5.Intervalo de 30 minutos;

6.Tonometria de aplanação com o mesmo tonômetro e pelo mesmo examinador;

7.Intervalo de 15 minutos (45 minutos após segunda gota);

8.Tonometria de aplanação com o mesmo tonômetro e pelo mesmo examinador;

9.Intervalo de 15 minutos (60 minutos após segunda gota);

10.Tonometria de aplanação com o mesmo tonômetro e pelo mesmo examinador. 
Tabela 1

Idade dos pacientes e medidas da Pio média do teste da ibopamina para OD

\begin{tabular}{lccccc}
\hline & & & \multicolumn{3}{c}{ Pio média OD Pio média OD Pio média OD } \\
& $\begin{array}{c}\text { Ida'após } \\
\text { (anos) }\end{array}$ & $\begin{array}{c}\text { 45'após } \\
\text { (mmH'após } \\
\text { (média OD }\end{array}$ & $\begin{array}{c}\text { ibopamina } \\
\text { (mmHg) }\end{array}$ & $\begin{array}{c}\text { ibopamina } \\
\text { (mmHg) }\end{array}$ & $\begin{array}{c}\text { ibopamina } \\
\text { (mmHg) }\end{array}$ \\
\hline Grupo 1 & 62,30 & 18,00 & 24,10 & 22,80 & 21,70 \\
Grupo 2 & 50,70 & 16,20 & 19,20 & 18,30 & 18,10 \\
Grupo 3 & 60,70 & 15,50 & 17,50 & 17,00 & 15,50 \\
\hline
\end{tabular}

Tabela 2

Idade dos pacientes e medidas da Pio média do teste da ibopamina para OE

\begin{tabular}{lccccc}
\hline & & & \multicolumn{3}{c}{ Pio média OE Pio média OE } \\
& $\begin{array}{c}\text { 30'após } \\
\text { Idade média média OE } \\
\text { (anos) }\end{array}$ & $\begin{array}{c}\text { Pio média OE } \\
\text { (mmHg) }\end{array}$ & $\begin{array}{c}\text { ibopamina } \\
\text { (mmHg) }\end{array}$ & $\begin{array}{c}\text { ibopamina } \\
\text { (mmHg) }\end{array}$ & $\begin{array}{c}\text { 60'após } \\
\text { ibopamina } \\
\text { (mmHg) }\end{array}$ \\
\hline Grupo1 & 62,30 & 17,50 & 22,60 & 22,20 & 21,60 \\
Grupo2 & 50,70 & 16,10 & 17,80 & 17,50 & 18,10 \\
Grupo3 & 60,70 & 15,40 & 17,40 & 16,70 & 18,43 \\
\hline
\end{tabular}

Tabela 3

Dados sobre a positividade do teste da ibopamina nos diferentes grupos

\begin{tabular}{lcccc}
\hline & Grupo 1 & Grupo 2 & Grupo 3 & Total \\
\hline Positivos & 16 & 9 & 6 & 31 \\
Negativos & 4 & 11 & 14 & 29 \\
Total & 20 & 20 & 20 & \\
\hline
\end{tabular}

O teste da ibopamina foi considerado positivo quando o aumento da Pio, em qualquer uma das medidas após a instilação, foi igual ou superior a $4 \mathrm{mmHg}$ em relação a Pio inicial.

Todos os colírios de ibopamina foram manipulados pela mesma farmácia de manipulação (Ophtalmos) e pelo mesmo profissional.

\section{$\underline{\text { RESULTADOS }}$}

O teste da Ibopamina foi positivo em $16(80 \%)$ olhos com GPAA (grupo 1), 9 (45\%) olhos suspeitos (grupo 2) e 6 (30\%) dos olhos considerados normais (grupo 3).

Todos os pacientes apresentaram midríase ( $>6 \mathrm{~mm})$ e queixaram-se de ardência após a instilação do colírio de ibopamina a $2 \%$. Nenhum efeito adverso sistêmico foi observado e/ou atribuído à ibopamina.

Os dados foram analisados utilizando-se o programa estatístico SPSS em sua versão 13.0.

Adotamos o nível de significância de 5\% $(0,005)$ para aplicação dos testes estatísticos, ou seja, quando o valor da significância calculada (p) for menor que 5\%, observamos uma diferença dita "estatisticamente significante". Não observamos diferenças estatisticamente significantes entre os grupos na análise das idades, bem como das pressões oculares basais bilateralmente. Nesta avaliação, foi aplicado o teste de KruskalWallis com o intuito de verificarmos possíveis diferenças entre os três grupos quando comparados concomitantemente.

Após a utilização da ibopamina tópica, porém, observamos que os grupos passaram a se comportar de maneira diferente. Houve significância estatística nesta divergência em suas avaliações nos minutos 30,45 e 60, 
tanto no olho direito quanto no esquerdo.Os grupos apresentaram-se mais diferentes entre si nas medidas da Pio após 45 e 60 minutos da utilização da ibopamina tópica em relação à medida após 30 minutos, com valor de $\mathrm{p}$ no olho esquerdo variando de 0,014 (minuto 30 ) para 0,003 (minuto 60) e 0,041 (minuto 30) para 0,009.

Utilizamos o teste de Mann-Whitney para identificarmos quais grupos diferem entre si para aquelas variáveis que se demonstraram estatisticamente significantes, ou seja, comportamentos diferentes na análise concomitante.

$\mathrm{Na}$ avaliação dos grupos controle $\mathrm{x}$ suspeito observamos apenas comportamentos diferentes $(p=0,020)$ na variável minuto 60 para olho esquerdo. Já na avaliação pareada dos grupos controle x glaucomatoso, houve diferença estatisticamente significante em todas as variáveis (minutos 30, 45 e 60) bilateralmente, sendo esta análise demonstrativa que a elevação da Pio varia de maneira mais significativa nos portadores de glaucoma. A avaliação dos grupos suspeitos $\mathrm{x}$ glaucomatosos apresentou comportamento intermediário em relação às anteriores, com diferenças estatisticamente significantes no minuto 30 para o olho esquerdo $(\mathrm{p}=0,030)$ e no minuto 45 para o olho esquerdo $(\mathrm{p}=0,002)$.

Observamos, portanto, que os grupos controle e glaucomatoso apresentam-se como sendo os "mais diferentes" entre si após utilização da ibopamina e que os grupos controle e suspeito foram os que menos divergiram após a sua utilização. Com o intuito de verificarmos possíveis diferenças entre os quatro momentos de observação, quando comparados concomitantemente por grupo considerado, utilizamos o teste de Friedman. Nos grupos controle, suspeito e glaucomatoso foi obtida diferença estatística, ou seja, dentro de cada grupo houve diferença de comportamento da Pio nas medidas basal, 30, 45 e 60 minutos.

Como em todas as comparações realizadas observamos diferenças estatisticamente significantes, utilizamos o Teste dos Postos Sinalizados de Wilcoxon para identificarmos os momentos que se diferenciam entre si. $\mathrm{Na}$ avaliação do grupo controle, observamos que o "basal" diferencia-se do momento "30",o momento "30" diferencia-se do momento " 60 ", e o momento " 45 " diferencia-se do momento "60", para ambos os lados. Na avaliação do grupo suspeito e glaucomatoso, no geral, o momento "basal" diferencia-se dos demais momentos que, por sua vez, são estatisticamente semelhantes. Para identificarmos valores de "notas de corte" ou "cutoffs" para o par de grupos controle e glaucomatoso utilizamos a análise da curva ROC considerando os lados e momentos de observação. A melhor relação sensibilida- de/especificidade para o teste, tanto para o olho direito quanto para o olho esquerdo foi observada no momento (minuto) 60, atingindo área sob curva de 0,90 bilateralmente. A nota de corte correspondente ao minuto 60 para o olho direito encontrada foi 17,00 , indicando valor de sensibilidade e especificidade do teste de $90 \%$.A nota de corte correspondente ao minuto 60 para o olho esquerdo encontrada foi de 16,50, indicando valor de sensibilidade e especificidade de $90 \%$. Para os diversos momentos de observação, valores de Pio menores a cada nota de corte correspondente indicam maior probabilidade de pertinência ao grupo controle, enquanto valores maiores do que a nota de corte, indicam maior probabilidade de pertinência ao grupo glaucomatoso.

\section{DıscussÃo}

A ibopamina (N-metildopamina 3,4 ester diisobutírico) utilizada como colírio é hidrolizada à epinina, um análogo da dopamina quando em contato com meio ocular. A epinina combina-se com receptores alpha adrenérgicos e D1 dopaminérgicos que causam,respectivamente, após sua ativação, midríase não cicloplégica e aumento na produção de humor aquoso. Devido à deficiente drenagem intraocular presente em indivíduos glaucomatosos, estes pacientes apresentam aumento da Pio significativo quando submetidos à aplicação de ibopamina tópica ${ }^{(2,5,7)}$. Seguindo este princípio, diversos testes foram estudados como indicadores prognósticos do glaucoma, como os testes de sobrecarga hídrica, provocativos de midríase e ensaios terapêuticos ${ }^{(8)}$.

Existem relatos na literatura demonstrando que a ibopamina tópica é capaz de identificar alteração no escoamento do humor aquoso mesmo em olhos com Pio estatisticamente normal $^{(3)}$, sendo porém este aumento mais evidente em olhos glaucomatosos, evidenciando a comprovada ação sobre o equilíbrio hidroeletrolítico ocular.

Utilizamos em nosso estudo a ibopamina a $2 \%$, bem como em outros ensaios descritos na literatura. Um estudo, comparando ibopamina tópica a 1 e $2 \%$ como teste provocativo para GPAA, encontrou a melhor relação sensibilidade/especificidade na observação da Pio após 45 minutos da administração de ibopamina a $2 \%$, porém não foi encontrada diferença estatisticamente significante em qualquer medida comparando as duas concentrações. Os autores recomendam a utilização da ibopamina a 2\% como teste provocativo por sua maior sensibilidade. A ibopamina a $1 \%$ é indicada para o tratamento da 
hipotonia ocular por sua menor concentração e também, boa relação sensibilidade/especificidade ${ }^{(7,9)}$.

Em nosso estudo, todos os pacientes relataram ardência de leve intensidade após aplicação do colírio, queixa esta frequente nos relatos da literatura. Alguns ${ }^{(3)}$ pacientes queixaram-se do odor desagradável do colírio, sendo esta queixa pouco frequente em estudos semelhantes.

Utilizamos 2 gotas de ibopamina a $\%$ com intervalo de 5 minutos entre cada aplicação, bem como relatos prévios da literatura ${ }^{(7,8,10)}$ e consideramos o teste positivo quando observado aumento igual ou maior a $4 \mathrm{mmHg}$ em relação a Pio basal, o que é descrito na grande maioria dos relatos, embora alguns estudos considerem um aumento maior ou igual a $3 \mathrm{mmHg}$ como positivo ${ }^{(7)}$.

Utilizamos ibopamina em pacientes glaucomatosos em uso ou não de medicação hipotensora, exceto os análogos de prostaglandinas, devido ao estudo na literatura que observou comportamento semelhante das pressões ao teste com diferentes drogas hipotensoras, o que não foi observado nos usuários de análogos de prostaglandinas. No estudo essa diferença é atribuída hipoteticamente à drenagem alternativa pela via úveo-escleral ${ }^{(8.11)}$.

Realizamos aferição da Pio em 30, 45 e 60 minutos, como descrito na grande maioria dos estudos. Um estudo $^{(8)}$ realizou a medida em 180 minutos, o que não concordamos, considerando tempo excessivo dispendido pelo paciente e metabolização de grande parte da ibopamina, perdendo assim importância para real aplicação do teste no ambulatório oftalmológico.

Em nosso estudo, os grupos controle e glaucomatosos foram os que demonstraram comportamentos mais diferentes entre si, com maior elevação da Pio em glaucomatosos.

Observamos também que dentro de cada grupo, o momento de observação (30, 45 ou 60) gera valores estatisticamente diferentes entre si, sendo a principal variação nas comparações das medidas após a administração da ibopamina com a Pio basal e não propriamente com os momentos de observação entre si.

Um estudo comparativo da Pio em 30, 60 e 180 minutos observou um aumento estatisticamente maior no minuto 30 , com sensibilidade de $87 \%$ e especificidade de $95 \%{ }^{(8)}$.

Já na avaliação de Magacho et al. ${ }^{(7)}$ o teste demonstrou melhores resultados no minuto 45 para ambos os olhos com sensibilidade de $84,6 \%$ e especificidade de 73,3\%.

Em nossa avaliação, encontramos melhor eficiência do teste no minuto 60 , bilateralmente, atingindo valores de sensibilidade e especificidade de $90 \%$.

\section{$\underline{\text { ConClusÃo }}$}

O teste provocativo da ibopamina a $2 \%$ pode ser utilizado com segurança na propedêutica do GPAA. A ibopamina gera maiores alterações na Pio em pacientes glaucomatosos, alcançando maior sensibilidade e especificidade como teste diagnóstico quando a Pio é medida 60 minutos após sua aplicação.

\section{Abstract}

Purpose: Evaluation of the effects of topic ibopamine in normal eyes, suspects and eyes with primary open-angle glaucoma (POAG) as well as your efficacy as a provocative test for glaucoma. Methods: 10 POAG pacients (20 eyes), 10 pacients suspects of POAG (20 eyes) and 10 normal Pacients (20 eyes) were selected and submitted to the topic ibopamine test. The test was considered positive when in one of the measures (30, 45 and 60 minutes) after instillation of 2 drops of $2 \%$ ibopamine, 5 minutes apart, the intraocular pressure (IOP) elevation was $=4 \mathrm{mmHg}$. Results: The ibopamine test was positive in $16(88 \%)$ eyes with POAG, $9(45 \%)$ eyes suspects of POAG and $6(30 \%)$ normal eyes. The best sensitivity/specificity ratio was achieved at 60 minutes for both eyes (Se/Sp:90\%). All patients described a slight burning after ibopamine's instillation. Conclusion: The results of this study suggests that topical $2 \%$ ibopamine is a secure alternative to evaluate outflow system impairment in eyes with glaucoma.

Keywords: Dopamine agonists/administration \& dosage; Open-angle glaucoma/diagnosis; Intraocular pressure/drug effects; Ophthalmic solutions; Sensitivity and specificity

\section{$\underline{\text { RefERÊNCIAS }}$}

1. Virno M, Taverniti L, Taloni M, Ioppolo A, Pellegrino N. Studio sperimentale degli effetti locali e sistemici dell'ibopamina, farmaco dotato di attivitá adrenergica e dopaminergica. Boll Ocul. 1986;65:1169

2. Virno M, Taverniti L, Motolese E, Taloni M, Bruni P, PecoriGiraldi J. Ibopamina: nuovo midriatico non cicloplegico (nota preliminare). Boll Ocul. 1986;65:1135-46

3. Virno M, Pecori-Giraldi J, Taverniti L, Taloni M, Pannarale L. Intraocular hypertensive effects of topically administered ibopamine in eyes with hydrodynamic disorders: provocative test for glaucoma. Glaucoma. 1990;12:88-92.

4. Virno M, Gazzaniga A, Taverniti L, Pecori Giraldi J, De Gregorio F. Dopamine, dopaminergic drugs and ocular hypertension. Int Ophthalmol. 1992;16(4-5):349-53. 
5. Virno M, Taverniti L, De Gregorio F, Sedran L, Longo F. Increase in aqueous humor production following D1 receptors activation by means of ibopamine. Int Ophthalmol. 19961997;20(1-3):141-6.

6. Virno M, Pannarale L, Arrico L, DeGregorio F. Increase in aqueous humor production by topical administration of ibopamine, a dopaminergic agonist, in glaucomatous and healthy eyes. Invest Ophthalmol. 1994;:2052. [The Association for Research in Vision and Ophthalmology Annual Meeting. Sarasota, Florida, May 1-6, 1994].

7. Magacho L, Costa ML, Dessimoni A, Avila MP. Comparação entre o teste de ibopamina a 1 por cento e a 2 por cento no glaucoma primário de ângulo aberto: sensibilidade, especificidade e tolerabilidade. Arq Bras Oftalmol. 2006;69(5):695-9.

8. Magacho L, Costa ML, Lima FE, Magacho B, Ávila MP. Análogos das prostaglandinas diminuem a sensibilidade do teste provocativo da ibopamina no glaucoma. Arq Bras Oftalmol. 2006;69(2):193-6.
9. Lima FE, Pacífico M, Carvalho D, Avila M. Ibopamina tópica em hipotensão ocular. Rev Bras Oftalmol. 1999;58(2):143-7.

10. Lima FE, Guimarães NLD, Santos LM, Costa LP, Ávila M. Ibopamina tópica na propedêutica do glaucoma. Rev Bras Oftalmol. 2002;61(2):109-13.

11. Magacho L, Lima FE, Costa ML, Fayad FA, Guimarães NL, Avila MP. Ibopamine provocative test and glaucoma: consideration of factors that may influence the examination. Curr Eye Res. 2004;28(3):189-93.

\section{Endereço para correspondência: Marina Lotto Cordeiro \\ Rua das Aroeiras, $\mathrm{n}^{\circ}$ 392- apto 101 \\ CEP 09090-000 - Santo André (SP), Brasil \\ E-mail: cordeiromarina@hotmail}

